\title{
Quantum mechanics and much more
}

\section{Alisa Bokulich: Reexamining the quantum-classical relation. Beyond reductionism and pluralism. Cambridge: Cambridge University Press, 2008, x+95pp, \$74 HB}

\section{Dennis Dieks}

Published online: 22 October 2010

(C) The Author(s) 2010. This article is published with open access at Springerlink.com

At bottom, the aim of this book is the formulation and defence of a novel philosophical stance concerning the general relation between successor theories in science. But its method is inductive: almost the entire book is devoted to one detailed case study, the relation between classical mechanics and quantum mechanics. The author, Alisa Bokulich, dwells extensively on the history of quantum mechanics and the philosophical ideas of its founding fathers and also on recent developments in physics. The book has thus become a goldmine of information and original ideas about quantum mechanics and its philosophy, in addition to being a philosophical treatise about the relation between theories.

The first chapters are taken up by a consideration and comparison of the philosophical views of Heisenberg, Dirac and Bohr. Heisenberg's conception of "closed theories", with classical mechanics as a prime example of such a theory, has many traits in common with Kuhn's analysis in terms of paradigms and is also close to Nancy Cartwright's theoretical pluralism. By contrast, Dirac regarded quantum mechanics not as a completely new conceptual scheme but rather as a generalization of classical mechanics, in which central structural features have been preserved. Bohr also spoke about correspondences between classical and quantum mechanics and even made a principle out of this (the Correspondence Principle). Bohr notoriously added that classical mechanics is an indispensable conceptual precondition for quantum mechanics: according to him, the formulation of quantum theory is only possible on the basis of the language of classical theory. In her discussion of Bohr's ideas, Bokulich proposes and defends an interesting new interpretation of the Correspondence Principle. According to her analysis, the correspondence meant by Bohr is not the empirical equivalence of the two theories in limiting situations (the limit of large quantum numbers, for example). Rather,

D. Dieks $(\bowtie)$

History and Foundations of Science, Utrecht University, POB 80010, 3508 TA Utrecht, The Netherlands e-mail: d.dieks@uu.nl 
says Bokulich, it was Bohr's intention to point out that there exist general structural relations between classical and quantum theory, quite independently of any limit. This theme of structural continuity between theories, without the consideration of limiting situations, constitutes the Leitmotiv of the book's philosophical program.

A central chapter of the book, "Semiclassical mechanics: Putting quantum flesh on classical bones", is devoted to the concrete elaboration of this continuity theme for the case of quantum mechanics as seen from a modern perspective. Semiclassical mechanics is the name of a relatively new discipline in physics: it is a field halfway between quantum and classical mechanics, in which classical concepts like particle trajectories are used to deal with quantum phenomena. Obviously, quantum principles must enter the theoretical treatment somewhere, so that there can be no question of a purely classical approach. But it turns out that it is nevertheless often possible to use classical concepts (which according to fundamental quantum theory do not refer to anything objectively existing in the world) complemented by some simple quantum principles in order to arrive at easily understandable and visualizable ways of solving quantum problems. Even more striking is the fact that these classical concepts sometimes have a direct relation to what is found in experiments: for example, in some cases, classical trajectories can almost be "seen" directly in the data, in spite of the fact that these trajectories are pure fictions from the point of view of "official" quantum theory. The standard way of interpreting this is in terms of limiting cases and approximations: classical concepts become approximately applicable in the classical limit of quantum theory. But Bokulich opposes this standard viewpoint. What is rather at stake, she says, is the exploitation of structural continuities between quantum and classical theory full stop, without any approximations and limits. There is a lot of detailed and interesting physics in this chapter, with particular emphasis on cases of "quantum chaos" (in which the classical counterpart of a quantum system exhibits chaotic behaviour, whereas strictly speaking there is no chaos from a rigorous quantum point of view).

In line with the idea that classical structures in quantum mechanics are more fundamental than suggested by the standard "approximations view", Bokulich next argues that such classical structures not only can be employed to formulate efficient pragmatic procedures to get a grip on quantum phenomena but can also genuinely explain them. Even more, she claims that reasoning in terms of classical particle trajectories in many cases yields an explanation of quantum phenomena that is deeper than what can be furnished by a full quantum mechanical calculation. This evidently raises several questions, not the least of which is how concepts that according to our best theory (i.e. quantum mechanics) do not refer to anything objectively existing at all, can possibly explain. In reply, Bokulich introduces the concept of "structural model explanations". These are explanatory arguments which exploit structural theoretical dependencies, without the assumption that the elements that figure in these dependencies refer directly to things/processes in physical reality. In addition, she argues that one of the main purposes of explanations is to give understanding and that arguments using a picture with classical trajectories may lead to more and deeper understanding than a formal quantum mechanical calculation. This, of course, raises the question of what understanding consists in, 
and what the criterion for better understanding is. On this point, Bokulich appeals to a proposal by Hitchcock and Woodward (Nous 37, 2003, 181-199), according to which explanations are deeper if they are able to answer a greater range of "whatif" questions. To me, however, it seems clear that this idea is inadequate here: without doubt, precise quantum mechanical calculations can answer many more such questions than any semiclassical argument, so it would follow that quantum mechanics always gives more understanding than pictures using particle trajectories. What appears to be missing here is a consideration of pragmatic aspects of explanation. Indeed, the semiclassical treatment yields a practically manageable, intuitive picture with the help of which essential features of the situation can easily be recognized; and it is in this sense that it provides better understanding than an austere quantum mechanical calculation. An analysis of scientific understanding along these lines, as given by De Regt and Dieks (Synthese 144, 2005, 137-170) seems to fit better in Bokulich's program.

The consideration of quantum and classical mechanics is meant as a stepping stone towards the development of a general account of the relation between successor theories in science, as Alisa Bokulich makes clear at the end of her book. The central idea of this general position is that the history of science should not be regarded as a succession of incommensurable paradigms or closed theories, but rather as a process of generalization in which essential structural features are preserved. This "interstructuralism", as she baptizes the idea, is more akin to the ideas of Dirac than those of Heisenberg or Bohr (the latter puts too much weight on the indispensability of classical language). But, as Bokulich admits, much work remains to be done to work out this position. For example, a precise characterization of structural identity or similarity would be welcome: in spite of the details about semiclassical physics, it remains a bit vague in what exact sense classical trajectories are parts of the structure of quantum mechanics. Another natural question, also indicated by Bokulich, is how interstructuralism relates to the ongoing debate about structural realism. And, of course, one may wonder whether the situation in quantum mechanics effortlessly generalizes to other cases. These are only some of the stimulating questions raised by Alisa Bokulich's work. I think the book is an important contribution both to the history and philosophy of quantum mechanics and to the general philosophy of science.

Open Access This article is distributed under the terms of the Creative Commons Attribution Noncommercial License which permits any noncommercial use, distribution, and reproduction in any medium, provided the original author(s) and source are credited. 